ARTICLE

\title{
Crystal structure and functional implication of bacterial STING
}

\author{
Tzu-Ping Ko (1) 1,7, Yu-Chuan Wang 2,7, Chia-Shin Yang 2,7, Mei-Hui Hou ${ }^{2,7}$, Chao-Jung Chen (i) 3,4, \\ Yi-Fang Chiu ${ }^{2} \&$ Yeh Chen (iD) 2,5,6凶
}

Mammalian innate immune sensor STING (STimulator of INterferon Gene) was recently found to originate from bacteria. During phage infection, bacterial STING sense c-di-GMP generated by the CD-NTase (cGAS/DncV-like nucleotidyltransferase) encoded in the same operon and signal suicide commitment as a defense strategy that restricts phage propagation. However, the precise binding mode of c-di-GMP to bacterial STING and the specific recognition mechanism are still elusive. Here, we determine two complex crystal structures of bacterial STING/c-di-GMP, which provide a clear picture of how c-di-GMP is distinguished from other cyclic dinucleotides. The protein-protein interactions further reveal the driving force behind filament formation of bacterial STING. Finally, we group the bacterial STING into two classes based on the conserved motif in $\beta$-strand lid, which dictate their ligand specificity and oligomerization mechanism, and propose an evolution-based model that describes the transition from c-di-GMP-dependent signaling in bacteria to $2^{\prime} 3^{\prime}$-cGAMP-dependent signaling in eukaryotes.

\footnotetext{
${ }^{1}$ Institute of Biological Chemistry, Academia Sinica, Taipei 115, Taiwan. ${ }^{2}$ Institute of New Drug Development, China Medical University, Taichung 406, Taiwan. ${ }^{3}$ Graduate Institute of Integrated Medicine, China Medical University, Taichung 406, Taiwan. ${ }^{4}$ Proteomics Core Laboratory, Department of Medical Research, China Medical University Hospital, Taichung 404, Taiwan. ${ }^{5}$ Research Center for Cancer Biology, China Medical University, Taichung 406, Taiwan. ${ }^{6}$ New Drug Development Center, China Medical University, Taichung 406, Taiwan. ${ }^{7}$ These authors contributed equally: Tzu-Ping Ko, Yu-Chuan Wang, Chia-Shin Yang, Mei-Hui Hou. 凶email: chyeah6599@mail.cmu.edu.tw
} 
$\mathrm{B}$ iological conflicts between different organisms and different kingdoms of life have been evolving since ancient time. The arm races never stop especially between bacteria and phages $^{1-4}$. For example, the anti-CRISPR (Clustered Regularly Interspaced Short Palindromic Repeats) proteins in phages combat against the CRISPR-cas system in bacteria ${ }^{5,6}$. The more recently discovered CBASS ${ }^{7,8}$ in the bacterial arsenal consists of a CD-NTase that synthesizes diverse cyclic di- and tri-nucleotide (CDN and CTN) second messengers upon phage infection and an effector protein that binds to the CD-NTase product and induces "programmed cell death" through its various activities ${ }^{7-9}$. In eukaryotes, the cGAS-STING pathway of innate immunity, akin to CBASS, plays critical roles in antiviral, anticancer, and autophagy mechanisms ${ }^{7,10-12}$. During viral infection, the sensor cGAS rapidly recognizes foreign nucleic acid molecules and synthesizes the second messenger 2'3'-cGAMP that activates downstream STING effectors, leading to the expression of interferon-related genes ${ }^{10}$.

A recent study demonstrated that STING proteins indeed originated in bacteria ${ }^{12}$. They are fused with transmembrane or Toll/Interleukin-1 receptor (TIR) domain that confers the antiphage activity by forming membrane pores or cleaving vital electron carrier $\mathrm{NAD}^{+} 7,13,14$. Morehouse et al. showed that during phage infection, bacterial STING proteins stringently recognize $c$-di-GMP generated by $\mathrm{CD}$-NTase and signal the infected bacteria to commit suicide as a defense strategy against phage propagation ${ }^{7,12}$. However, the reported 3',3'-cGAMP complex structure of Flavobacteriaceae sp. STING (FsSTING) does not distinguish the guanine base from adenine and the rationale for binding preference of c-di-GMP over 3',3'-cGAMP remains unclear ${ }^{12}$.

To provide additional information about cyclic dinucleotide recognition mechanism by bacterial STING proteins, we expressed, purified, crystallized, and determined the structures of two STING domains from Prevotella corporis (PcSTING, GenBank ID: KXA32418.1) and Myroides sp. ZB35 (MySTING, IMG Gene ID: 2719779365). The presence of STING-bound c-di-GMP in the crystal allowed detailed analysis of its specific binding mode. To obtain further insights into the function of bacterial STING protein, we separated and purified the oligomerized TIR-STING from the dimers and accessed their $\mathrm{NAD}^{+}$cleavage activity. In addition, the detailed oligomerization mechanism is also unveiled by the symmetry-related STING molecules in our crystal structures.

\section{Results}

Specific recognition of c-di-GMP by STING. Initial attempts to solve the PcSTING and MySTING structures by molecular replacement (MR) using the known models of FsSTING and Capnocytophaga granulosa STING (CgSTING) failed, suggesting significant differences between the bacterial STING domains ${ }^{12}$. We turned to prepare SeMet-labeled PcSTING and solved the structure at $2.25-\AA$ resolution (Supplementary Table 1 ). The structure of MySTING was then solved by MR using the refined model of PcSTING (Supplementary Table 2). Both PcSTING and MySTING crystals contained two nearly identical protomers with root-mean-square deviations (RMSDs) of $0.29 \AA$ and $0.64 \AA$ for 158 and 160 matched $C a$ pairs in the asymmetric unit, forming canonical V-shaped dimers of STING domain (Fig. 1a, b). The overall architectures between PcSTING and MySTING dimers are very similar with a RMSD of $1.09 \AA$ for 272 matched $\mathrm{C} \alpha$ pairs. Each protomer adopts a mixed $\alpha / \beta$ fold with a central five-stranded $\beta$-sheet surrounded by five $\alpha$-helices and a flanking $\beta$-ribbon (Fig. 1c). A previous study showed that the overall dimension of FsSTING is smaller than that of human STING
(hSTING) (Supplementary Fig. 1a, b) ${ }^{12}$. Our PcSTING and MySTING structures have similar but slightly extended conformation in comparison with FsSTING (Supplementary Fig. 1c, d). The dimers show RMSDs of 2.8-3.4 $\AA$ from those of FsSTING and CgSTING, the latter matching mainly on one protomer due to its more open ligand-free conformation. Similar to FsSTING, both PcSTING and MySTING dimers contain a bound CDN in the central nucleotide-binding pocket and adopt a closed conformation (Fig. 1a, b). However, the dispositions of helix $\alpha 2$ and loops $\beta 3-\beta 4$ and $\alpha 3-\alpha 4$ vary greatly, as well as the loop lengths of $\alpha 1-\beta 1$ and $\beta 2-\beta 3$ (Supplementary Fig. $2 a, b$ ). Despite the similar overall protein topology, PcSTING and MySTING monomer differs from hSTING by an RMSD of 3.1-3.4 $\AA$ for 134-139 matched Ca atoms (Supplementary Fig. $2 c, d)$. Instead of the four-stranded $\beta$-sheet lids in metazoan STING, bacterial STING forms a two-stranded $\beta$-sheet lid that covers the ligand-binding site. Furthermore, the C-terminal tail (CTT) domain and its preceding helix in mammalian STING are also absent in PcSTING and MySTING (Supplementary Fig. 2c, d) ${ }^{15}$.

Although no nucleotide was included in the crystallization solution, the strong electron-density unambiguously identified the bound CDN as c-di-GMP (Fig. 1d, e). LC-MS/MS analysis further confirmed the existence of c-di-GMP in the bacterial STING protein samples (Supplementary Fig. $3 a-c$ ). Its presence was most likely a result of tight binding and co-purification of the STING proteins with endogenous c-di-GMP from the expression host. The previous study has shown that bacterial STING strongly prefers c-di-GMP over other CDNs ${ }^{12}$. However, the structure of FsSTING in complex with 3'3'-cGAMP could not discriminate adenine base from guanine, and the proposed specificitydetermining residue D169 of FsSTING could not fully explain the specificity for c-di-GMP ${ }^{12}$. Here, our structures demonstrate that the symmetric recognition of single c-di-GMP by dimeric bacterial STING proteins is dictated by the guanidinium group of arginine residue (R233/R230 in PcSTING/MySTING) that recognizes the $\mathrm{O} 6$ and $\mathrm{N} 7$ atoms of guanine base, the backbone oxygen and amide group (H238/H235 in PcSTING/MySTING) that form three hydrogen bonds to the Watson-Crick edge of guanine base and the aspartate residue (D252/D251 in PcSTING/ MySTING) that binds to the $\mathrm{N} 2$ atom of guanine base (Figs. If and $2 \mathrm{a}, \mathrm{c}$ ). Two or three water molecules further stabilize the interaction by hydrogen bonding to the $\mathrm{N} 1$ and $\mathrm{N} 2$ positions of guanine base and the free 2'-OH of ribose within c-di-GMP in both PcSTING and MySTING structures (Fig. 2a, c). In addition to the specific $\mathrm{H}$-bonding interaction, each guanine base of $\mathrm{c}$-diGMP is sandwiched by extensive $\pi-\pi$ stacking interaction formed by F171 of one PcSTING protomer and Y235 of the other protomer (Fig. 2b). The planar guanidinium group of R233 also stacks with the aromatic ring of Y235, forming a unique Phe/ Guanine/Tyr/Arg four-layer stack buttressed by the four H-bond interactions between R233 and c-di-GMP (Fig. 2b). This distinct four-layer stack binding mode of c-di-GMP is also observed in the MySTING structure (Fig. 2d). Furthermore, the multiple sequence alignment of bacterial STING family proteins demonstrated that the identified specificity-determining arginine residues (R233/R230/R151 in PcSTING/MySTING/FsSTING) are conserved (Supplementary Fig. 4). By contrast, R153 in FsSTING (equivalent to Y235/Y233 in PcSTING/MySTING), which was proposed to mediate cation $-\pi$ stacking interaction with guanine base in the previous study, is not conserved and could be replaced by Phe or Tyr in other bacterial STING family proteins (Supplementary Fig. 4) ${ }^{12,16}$. Therefore, it is clear that the four-layer stacking interaction mediated by the binding motif (Phe/Leu)/Gua/(Tyr/Phe)/Arg (e.g., F171/Gua/Y235/R233 in PcSTING or L169/Gua/Y232/R230 in MySTING), together with 

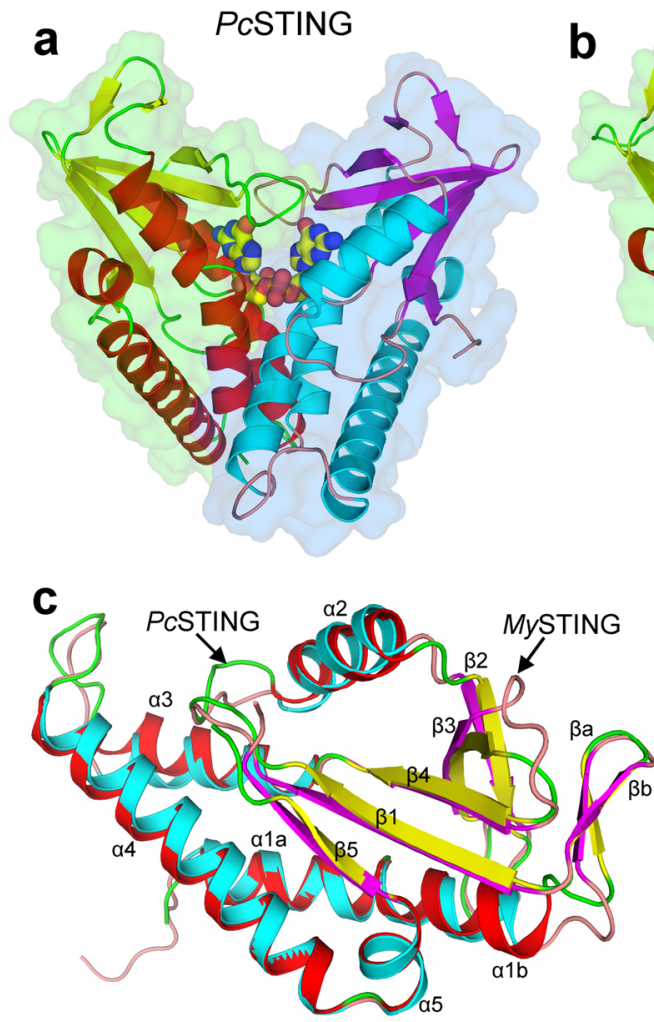
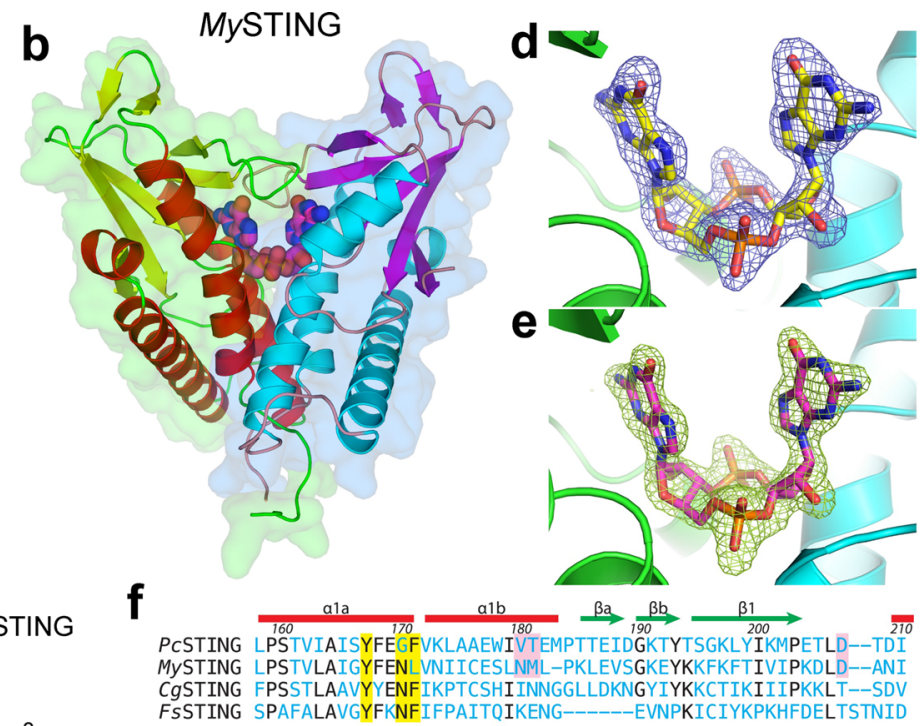

PCSTING LPSTVIATSYFEGFVVLAAEWIVTTEMPTTEIDGKTYTSGKLYIKMPETLD--TDI MYSTING LPSTVLAIGYFENLVNIICESLNML-PKLEVSGKEYKKFKFTIVIPKDLD--ANI
CgSTING FPSSTLAAVYYNFIKPTCSHIINNGGLLDKNGYIYKKCTIKIIIPKKLT--SDV FSSTING SPAFALAVGYFKNFIFPAITQIKENG------EVNPKICIYKPKHFDELTSTNID

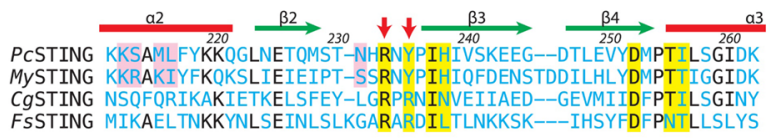

\begin{tabular}{c}
270 \\
\cline { 2 - 2 } PCSTING AIDMYFRVGHI-GKTTEQQLAEDNEMNNFKRVLQLLINEDSFCRECVEIL--
\end{tabular}

PCSTING AIDMYFRVGHI-GKTTEQQLAEDNEMNNFKRVLQLLINEDSFCRECVEIL-----
MYSTING AIEMFMRKGHI-GKTDQQKLLEERELRNFKTTLENLIATDAFAKEMVEVIIEE-MYSTING AIEMFMRKGHI-GKTDQQKLLEERELRNFKTTLENLIATDAFAKEMVEVIIEE--
CgSTING AISNLLPQDFN-SMSVDYEAILSRELERFVYTLKKIALRDGFDDLIKIVDEDN-CgSTING AISNLLPQDFN-SMSVDYEAILSRELERFVYTLKKIALRDGFDDLIKIVDEDN--
FSSTING YVDFKIASSNNNSSELKKKKFVELLIEQFYLKLNELIQENNLTNNITFCDKNLQG

Fig. 1 Crystal structures of bacterial STING in complex with c-di-GMP. Surface and cartoon models of dimeric assembly of (a) PCSTING and (b) MySTING are presented with the two protomers colored differently. The bound c-di-GMP molecule is shown as a space-filled model. c The PcSTING (colored red-green-yellow) and MySTING (cyan-pink-magenta) protomers are superimposed and shown as cartoon models with the five $\alpha$-helices and five $\beta$-strands indicated. d, e The Fo-Fc electron-density maps of the bound c-di-GMP molecules, each calculated by using the refined model with the ligand omitted, are contoured at 4- $\sigma$ level and shown as mesh for the (d) PCSTING and (e) MySTING crystals. f The amino acid sequences of four known bacterial STING proteins are aligned based on their 3D structures. Residues interacting with the bound CDNs are indicated by yellow shade. The conserved RXR or $\mathrm{RX}(\mathrm{Y} / \mathrm{F})$ motif in the $\beta$-strand lid which determines the ligand specificity is indicated by red arrows. The most involved residues in STING oligomerization as identified in this study are indicated by pink shade. CgSTING and FsSTING refer to the STING proteins from Capnocytophaga granulosa and Flavobacteriaceae sp.

the arginine residue (R233/R230 in PcSTING/MySTING) that recognizes the Hoogsteen edge of the guanine base, and the aspartate residue (D252/D251 in PcSTING/MySTING) that contacts the $\mathrm{N} 2$ position of the guanine base contributes to the stringent specificity for c-di-GMP by bacterial STING proteins. However, unlike PcSTING, the recognition of 3'3'-cGAMP by FsSTING is asymmetric (Fig. 2e). Inspection of the FsSTING structure revealed that the $3^{\prime} 3^{\prime}$-cGAMP and R153 adopt two different conformations each with about 0.5 occupancies (Fig. 2e) One R153 in FsSTING dimer form base-stacking interaction with guanine base to avoid the potential clash between the adenine base of 3'3'-cGAMP and the guanidine group of arginine while the other R153 in FsSTING dimer form two H-bonds with the guanine base (Fig. 2e). Superimposition of the structure of 3'3'cGAMP-FsSTING with c-di-GMP bound PcSTING or MySTING revealed that the differences in amino acid composition and tertiary structure of $\beta$-strand lids probably resulted in the differential recognition of cyclic dinucleotides between FsSTING and PcSTING/MySTING (Fig. 3a, b). The conserved specificitydetermining arginine residue R151 in FsSTING flipped away from the ligand-binding site, so the R153 in FsSTING structure could freely adopt two conformations to recognize 3'3'-cGAMP (Fig. 3a-d). By contrast, in PcSTING/MySTING the stacking interaction of R233/R230 and Y235/Y232 locks the conformation of the guanidinium group of R233/R230 into specific hydrogen bonds with the $\mathrm{O} 6$ and N7 atoms of c-di-GMP (Fig. 3a-d). To validate the importance of the specificity-determining tyrosine residue of the four-layer stack in c-di-GMP recognition, in vitro binding assays using isothermal titration calorimetry (ITC) were performed. Changing Y232 to arginine in MySTING indeed reduced the binding affinity to c-di-GMP by nearly 1200 -fold in comparison with the wild-type protein (Supplementary Fig. 5a, b). MySTING showed weak or no binding to 3'3'-cGAMP and cdi-AMP (Supplementary Fig. 5c, d).

Specificity for the symmetric [3'-5, $\left.3^{\prime}-5^{\prime}\right]$ phosphodiester bond linkage of $3^{\prime} 3^{\prime}$-c-di-GMP of bacteria STING is achieved by the side chain of conserved Thr residues (T255/T254 in PcSTING/ MySTING), Asn residues (N168 in MySTING) and the mainchain of I256/T255 in PcSTING/MySTING that form hydrogen bonds with phosphate backbone and the free 2'-OH of the ribose within c-di-GMP (Supplementary Fig. 6a, b). The symmetric ligand-recognition mode of bacterial STING thereby excludes its binding to the asymmetric mammalian cGAS product 2'3'cGAMP. Indeed, superimposition of complex structure of human STING-2'3'-cGAMP with PcSTING or MySTING structure shows that the free 3'-OH of 2'3'-cGAMP would cause serious steric hindrance to the side chain of I256/T255 in PcSTING/ MySTING, thus preventing it from binding (Supplementary Fig. 6c, d). Conversely, the intrinsic asymmetric dynamics of metazoan STINGs probably accounts for its preferential binding of 2'3'-cGAMP over other $3^{\prime} 3^{\prime}-\mathrm{CDNs}^{17-21}$. Previous studies have shown that binding of symmetric $3^{\prime} 3^{\prime}-c-d i-G M P$ can induce the formation of slightly asymmetric architecture of hSTING dimer, especially in $\beta$-strand lid ${ }^{17-20}$. In addition, the asymmetric 

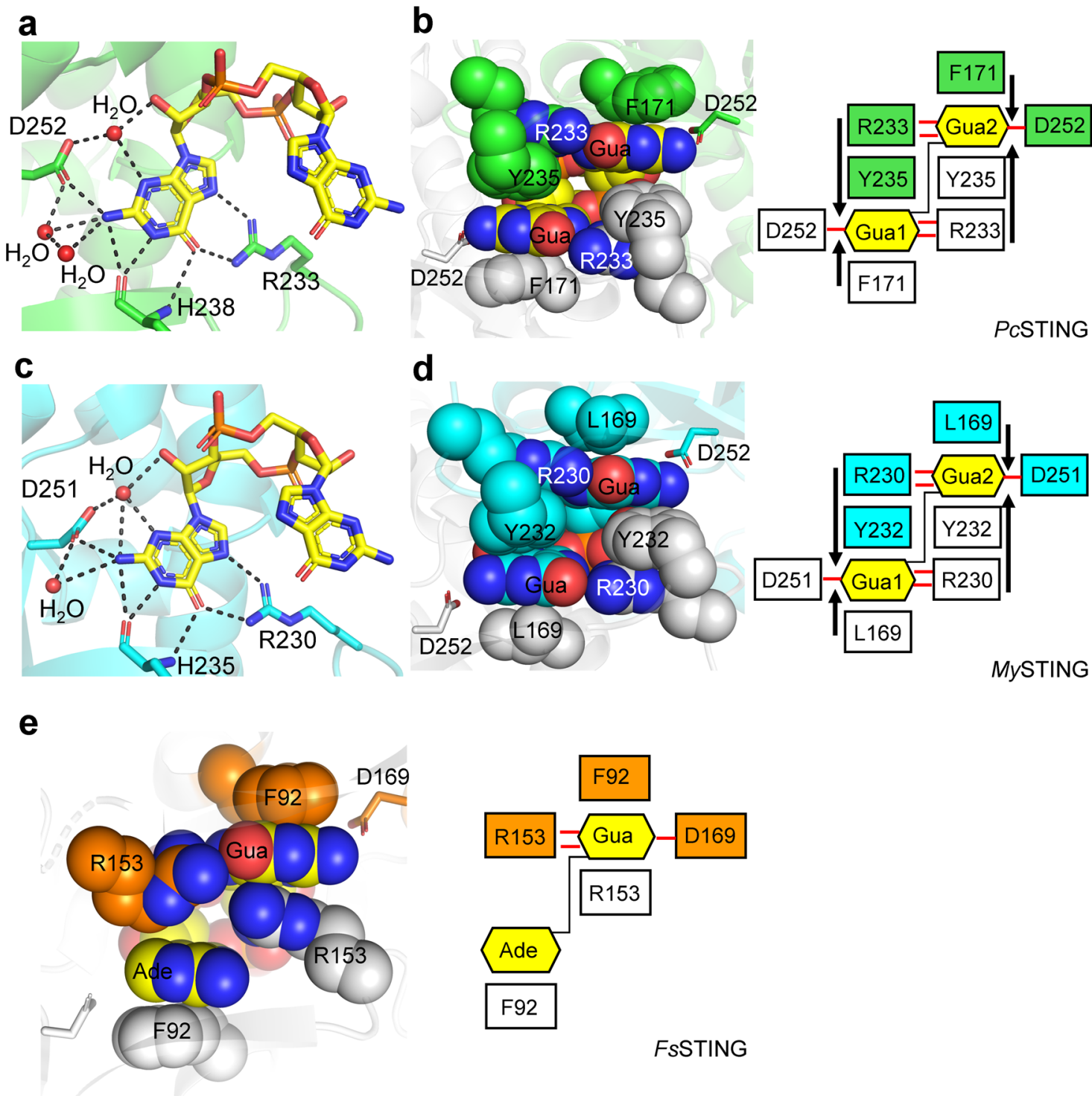

Fig. 2 Recognition of c-di-GMP by PcSTING/MySTING and 3'3'-cGAMP by FsSTING. a, c Enlarged view of the ligand-binding pocket of (a) PcSTING (green) and (c) MySTING (cyan). The hydrogen-bonding network that specifically recognizes the guanine nucleobase of c-di-GMP (yellow) are indicated by a black dashed line. The specificity-determining residues of PcSTING, MySTING, and c-di-GMP molecule are shown as sticks. Water molecules are shown as red spheres. b, d Left, the four-layer stacking interactions between c-di-GMP and (b) PcSTING dimer (green and light gray) and (d) MySTING dimer (cyan and light gray); right, schematic representation of the interactions. C-di-GMP and the residues involved in stacking (F171, R233, Y235 in PCSTING; L169, R230, Y232 in MySTING) are shown as spheres. One protomer is colored and the other is white. D252/D251 in PcSTING/MySTING that recognizes N2 of guanine base is shown as sticks. The four-layer stack binding mode is highlighted by black arrows. The $\mathrm{H}$-bonds are indicated by red lines. e Left, the stacking interactions between 3'3'-cGAMP (yellow) and FsSTING dimer (orange and light gray, PDB: 6WT4); right, schematic representation of the interactions. 3'3'-cGAMP and the residues involved in stacking (F92 and R153 in FsSTING) are shown as spheres. D169s in FsSTING are shown as sticks. Gua, guanine base; Ade, adenine base.

2'3'-cGAMP engaged well into the asymmetric ligand-binding pocket of porcine STING dimer, while other symmetric 3'3'CDNs could not fit well due to steric hindrance or lack of proper interactions $^{21}$. Furthermore, the conserved arginine residues R232 in the $\beta$-strand lids of hSTING make contact to the [2'-5', 3'-5'] phosphodiester bond linkage of 2'3'-cGAMP distinct from the base-specific recognition R233/R230 in PcSTING/MySTING (Fig. $3 \mathrm{e}, \mathrm{f})^{22}$. In summary, the structural data presented here fully explained the differential recognition of cyclic dinucleotides by bacterial STING family proteins and suggested that the symmetric/asymmetric ligand-recognition modes of STING proteins from different kingdoms are closely related to their specificity.
STING oligomerization and TIR activation. Morehouse et al. have shown that c-di-GMP induced the oligomerization of Sphingobacterium faecium TIR-STING (SfTIR-STING) into a long filament and activated the $\mathrm{NAD}^{+}$cleavage activity of its TIR domain ${ }^{12}$. However, the functional connection between the oligomerization and activation of TIR domain has not been established. Here, we present solid evidence that the filament formation of bacterial TIR-STING is directly linked to its NADase activation. We have separated and purified the oligomerized $M y$ TIR-STING proteins from the dimers and accessed their $\mathrm{NAD}^{+}$cleavage activity (Fig. 4a). The results demonstrate that without the addition of c-di-GMP, only oligomerized MyTIR-STING is active, which probably have incorporated 
a

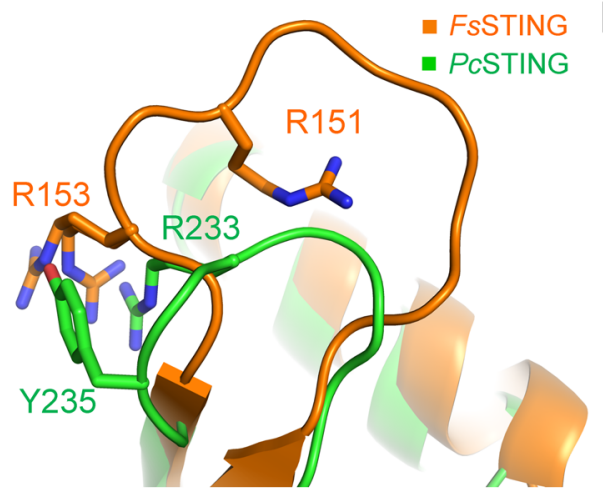

C

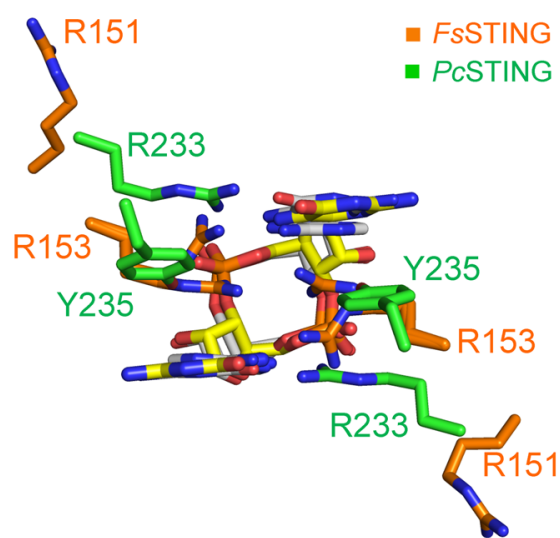

b

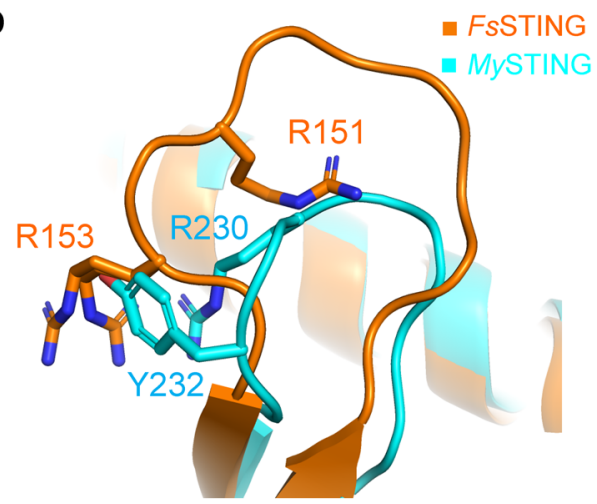

d

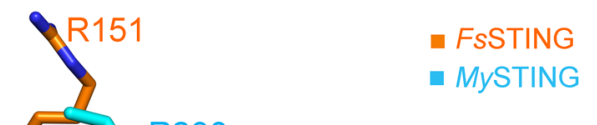

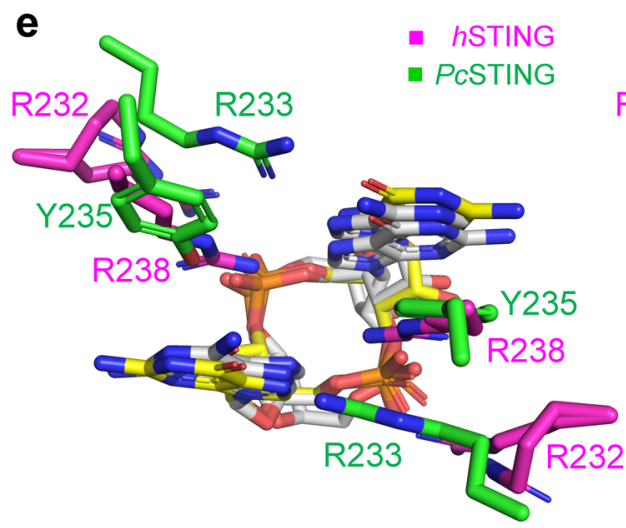

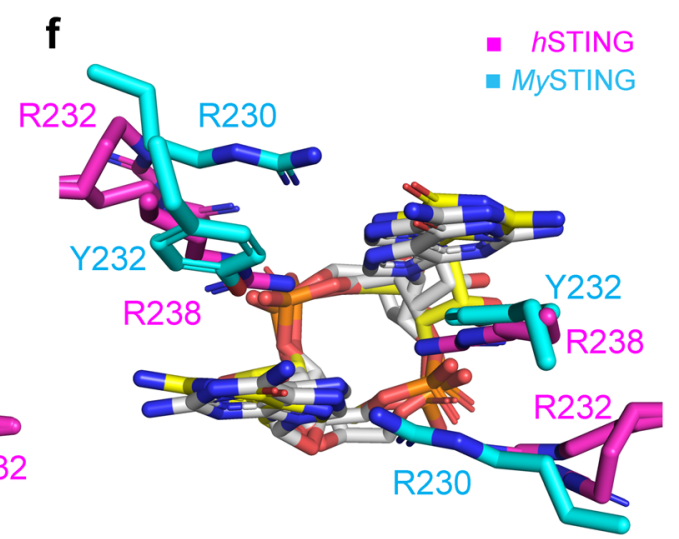

Fig. 3 Structural comparison of the $\boldsymbol{\beta}$-strand lids and the ligand-binding pockets of bacterial STING and metazoan STING. a, b Superimposition of the $\beta$ strand lids from the 3',3'-cGAMP-bound FsSTING (orange, PDB: 6WT4) and (a) PcSTING/c-di-GMP complex (green) or (b) MySTING/c-di-GMP complex (cyan). c, d Enlarged view of the ligand-binding pocket of FsSTING superimposed with (c) PCSTING or (d) MySTING. The specificity-determining residues in $\beta$-strand lids are shown as sticks and labeled. C-di-GMP and 3', $3^{\prime}$-cGAMP are shown as yellow and white sticks, respectively. e, $\mathbf{f}$ Enlarged views of the ligand-binding pockets following superimposition of the 2',3'-cGAMP-bound hSTING (magenta, PDB: 4KSY) with (e) PcSTING/c-di-GMP complex (green) or (f) MySTING/c-di-GMP complex (cyan). The specificity-determining residues in PcSTING, MySTING and hSTING are shown as sticks and labeled. C-diGMP and 2', 3'-cGAMP are shown as yellow and white sticks, respectively.

endogenous c-di-GMP from the E. coli host (Fig. 4b). Activation of dimeric MyTIR-STING requires the presence of c-di-GMP, which induces its oligomerization into long filaments (Fig. 4b). It has little effects on the already oligomerized MyTIR-STING proteins probably due to saturated c-di-GMP binding and imperfect assembly formation, whose NADase activity is also lower than that of freshly activated dimers. The activation effects of other CDNs for the TIR effector domain of bacterial STING proteins were also evaluated by this method. C-di-GMP strongly activated MyTIR-STING, whereas the addition of up to $100 \mu \mathrm{M}$ 3'3'-cGAMP, c-di-AMP, or c-di-UMP did not further increase its NADase activity (Fig. 4c and Supplementary Fig. 7a, b).
Activation of TIR-STING protein in CBASS immunity system leads to depletion of $\mathrm{NAD}^{+}$and so-called "abortive infections" that restrict bacterial phage reproduction ${ }^{7}$. Indeed, overexpression of wild-type PcTIR-STING or MyTIR-STING in E. coli cells resulted in significant growth inhibition (Fig. 4d, e). Moreover, mutating the specificity-determining residue D252 or R233 in PcTIR-STING relieved the growth inhibition, validating the importance of these two residues in recognition of c-di-GMP (Fig. 4d). In summary, these biochemical data further support the specific activation of the STING-containing CBASS immunity system by c-di-GMP and validate the essential roles of specificitydetermining residues identified in this study. 

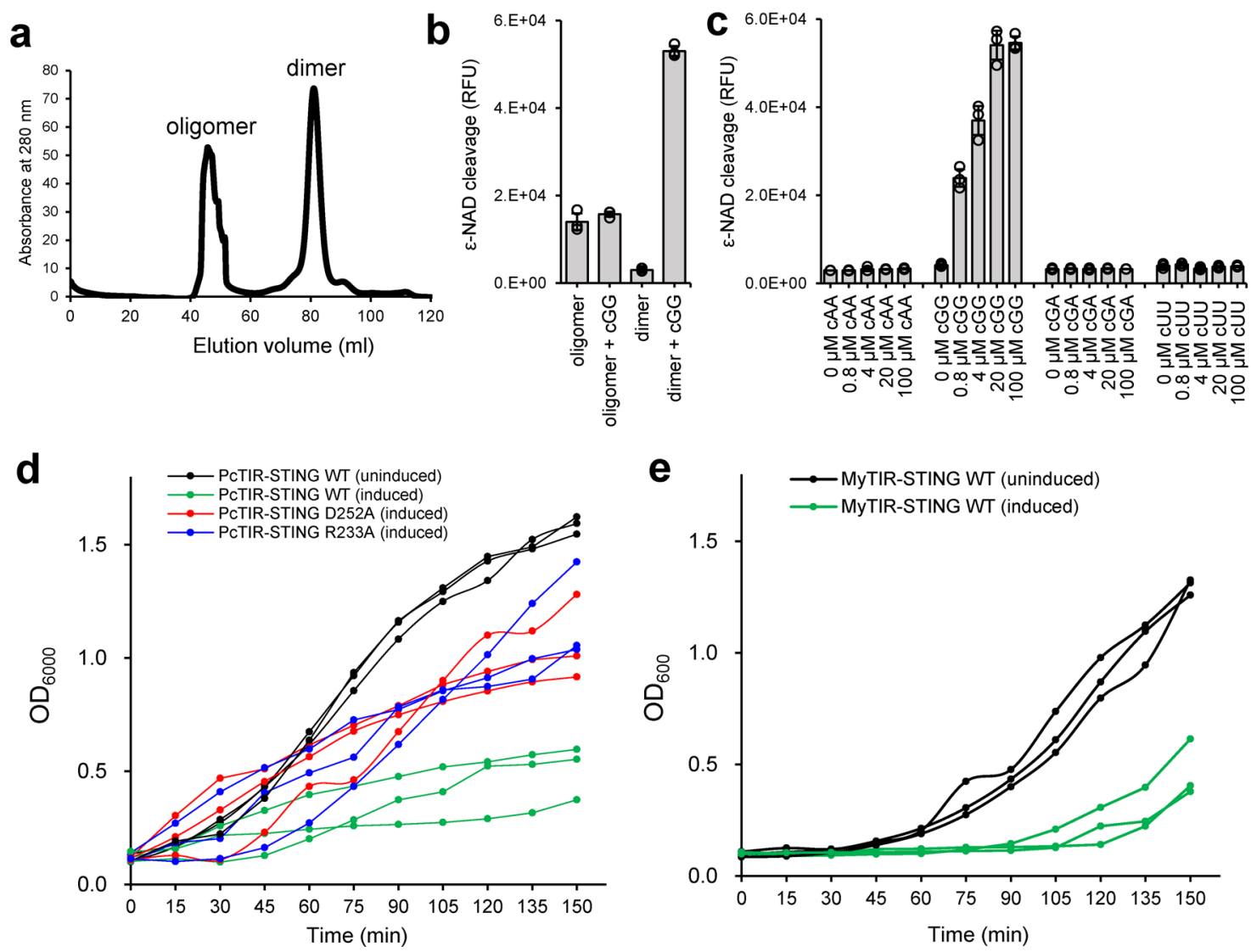

Fig. 4 Oligomerization and functional activation of bacterial TIR-STING. a Size-exclusion chromatography of MyTIR-STING proteins. The first peak eluting within void volume was denoted as oligomerized MyTIR-STING. The second peak was calculated as dimeric MyTIR-STING. b NAD+ cleavage activity of the oligomerized or dimerized MyTIR-STING in the presence or absence of $100 \mu \mathrm{M}$ c-di-GMP (cGG). c NAD ${ }^{+}$cleavage activity of dimerized MyTIR-STING with different cyclic dinucleotides $(0,0.8,4,20,100 \mu \mathrm{M})$. cAA, cyclic di-AMP; cGA, 3', $3^{\prime}$-cGAMP; cUU, c-di-UMP. The data in (b) and (c) are shown as mean \pm standard deviation for $n=3$ independent replicates. The effector TIR domain is active only in the presence of c-di-GMP. $\mathbf{d}$ Growth curves of E. coli cells overexpressing wild-type (green line), D252A (red line), or R233A (blue line) mutant PcTIR-STING proteins. The E. coli cells carrying wild-type PCTIR-STING without induction (black line) serve as a negative control. Significant bacterial growth inhibition was induced by wild-type PcTIRSTING proteins and largely restored by D252A or R233A mutant which abolish the specificity for c-di-GMP recognition. e MyTIR-STING toxicity analysis using E. coli cells producing endogenous c-di-GMP. Induction of the expression of MyTIR-STING proteins (green line) caused dramatic bacterial growth arrests in host cells compared with the no IPTG induction control (black line). The experiments in (d) and (e) were performed for $n=3$ biological replicates and each of them is shown.

Notably, the oligomerization state is implicated in the crystal structures of both PcSTING and MySTING. Each V-shaped dimer of PcSTING associates with its neighbors on both sides, which are symmetry-related by unit-cell translation along the $c$ axis (Fig. 5a, b). A similar arrangement of dimers along the $a$ axis of MySTING crystal suggests a common mode of dimer-dimer interaction (Fig. $5 \mathrm{c}, \mathrm{d}$ ). The dimer-dimer interface excludes $1440 \AA^{2}$ surface areas on the PcSTING dimer $\left(1420 \AA^{2}\right.$ on MySTING), comparable to the protomer-protomer interface of $1120 \AA^{2}\left(1500 \AA^{2}\right)$ upon dimer formation. If a continuous filament is formed in this way, each dimer will have $\sim 10 \%$ of its surface areas buried and at least 60 amino acid residues involved (Fig. 5e). The oligomerization of PcSTING and MySTING could be attributed to the highly complementary shape and the electrostatic interaction between one side of the PcSTING/MySTING protomer which is rich in positively charged residues and the other side of the PcSTING/MySTING protomer which contains a lot of negatively charged residues (Fig. 6a, b and Supplementary $8 \mathrm{a}, \mathrm{b}$ ). The extensive interfaces include the end of helix $\alpha 1$, the whole helix $\alpha 2$, the loop connecting helix $\alpha 3$ and $\alpha 4$, the end of helix $\alpha 4$, and helix $\alpha 5$ (Fig. 1c). The small helix $\alpha 5$ interacts with helix $\alpha 2$ of the other dimer by both hydrophobic and ionic interactions including two salt-bridges (K212-D301, K220-E306), one hydrogen bond (S213-S302), and the close sidechain packing of F303, K212, M215, and L216 (Fig. 6c). The same contacts are formed on the other side of PcSTING dimer (Fig. 6d), and similar interactions are also observed in the MySTING crystal. For example, the salt-bridging K209 and D300 (equivalent to K212 and D301 in PcSTING) together with E175 or an additional N171 contribute two or three ionic bonds that strengthen the filament formation of MySTING (Supplementary Fig. 8c, d). Moreover, the hydrophobic stacking interactions between the side chains of F302, K209, K212, and I213 are also conserved in MySTING (Fig. If and Supplementary Fig. 8c, d).

The loop connecting helix $\alpha 3$ and a4 (denoted 3-4 loop, residues 267-276) in PcSTING intercalated into a groove formed by helix $\alpha 1$ and $\alpha 4$ of protomer A' and the 3-4 loop of protomer $\mathrm{B}^{\prime}$ of the other dimer (Fig. 6e). The interactions are mediated mainly by the protein backbone with additional side-chain contacts of I273 and M266 (Fig. 6e). Similar extensive hydrophobic contacts are also observed in the 3-4 loop of MySTING, with the conserved I272 extended into the groove formed by helix $\alpha 1$ and $\alpha 4$ and M265 interacting with the 3-4 loop of protomer B' of the other dimer (Fig. If and Supplementary Fig. 8e). 
a
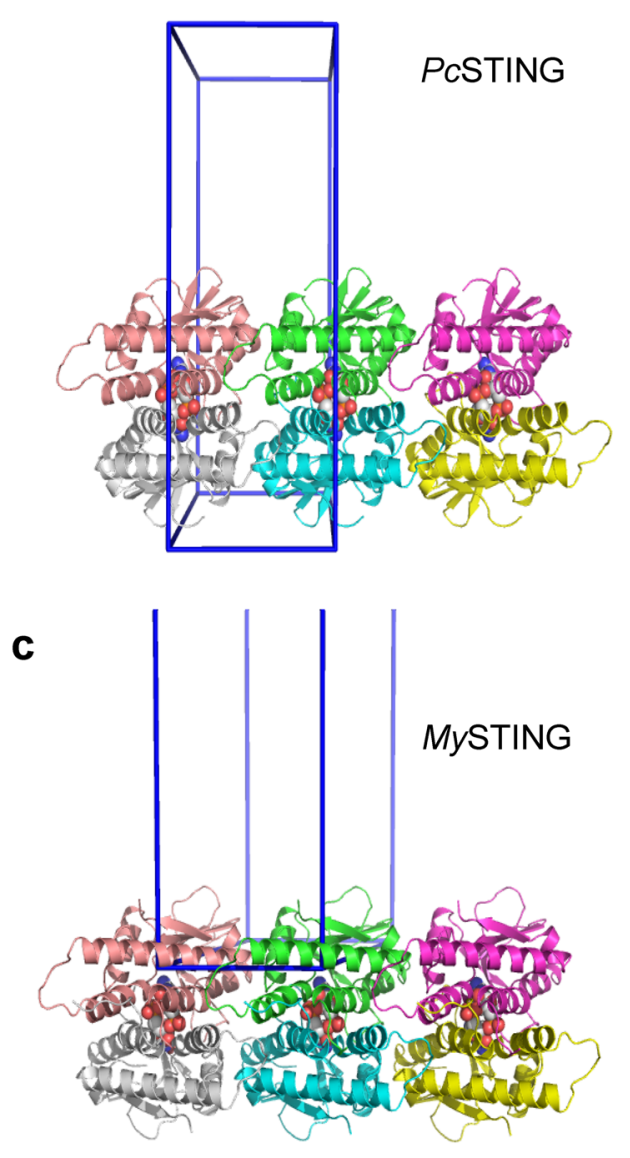

b

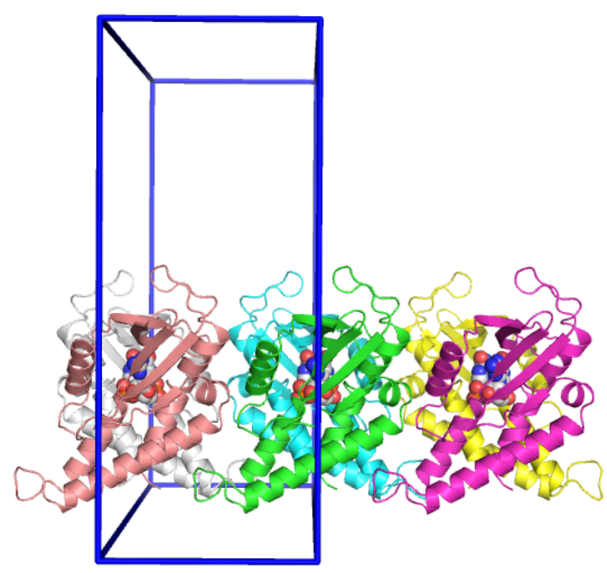

d

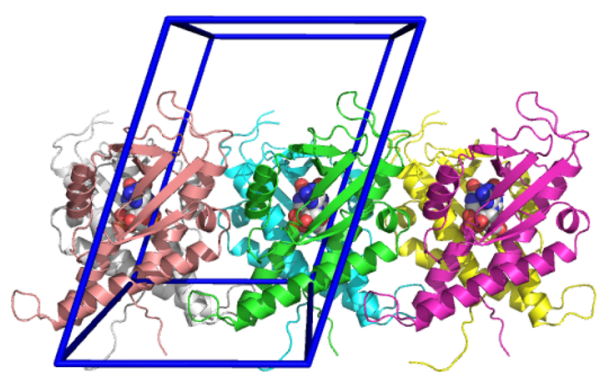

e

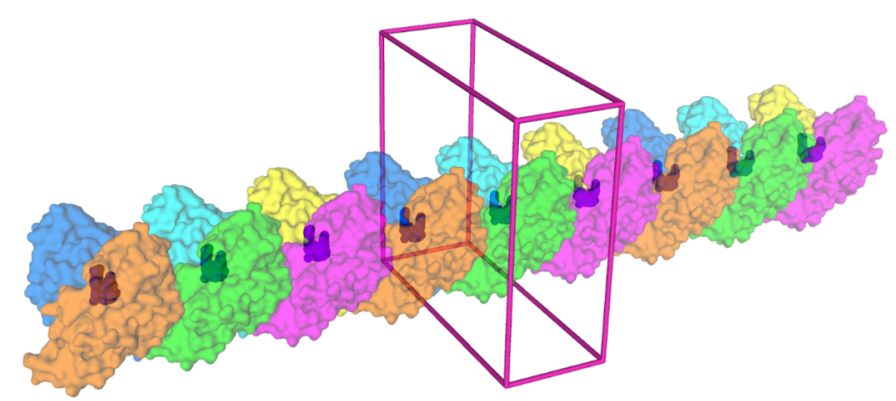

Fig. 5 Mode of bacterial STING oligomerization. a-d Two orthogonal views are shown with three PcSTING or MySTING dimers juxtaposed as seen in the crystals. The protomers are represented by ribbon models in different colors. The bound c-di-GMP molecules are shown as ball models. The unit cells are depicted as blue cages. In (a) and (b), the PCSTING dimers are viewed along the crystallographic $b$ - or $a$ axis, both with the $c$ axis lying horizontally. In (c) and (d), the MySTING dimers are viewed with the b-axis lying vertical or pointing upward. Both views of (c) and (d) have the a axis lying horizontal, and the MySTING dimers are arranged in a similar way as the PCSTING dimers in (a) and (b). e An extended packing diagram for possible filament formation of PCSTING dimers is shown with the protomers in alternatively different colors. The bound ligands are also presented by using ball models in blue, which can be seen through the translucent surface diagram of protein models. The unit cell is in magenta.

In summary, the crystal structures of PcSTING and MySTING clearly demonstrate that the interaction of helix $\alpha 2$ with the end of helix $\alpha 4$ and an additional small helix $\alpha 5$ together with the extensive hydrophobic contacts made by the 3-4 loop lock the dimer-to-dimer interface to form a continuous filament. A previous study predicted that the beginning of helix $\alpha 2$, the end of helix a4, and the 3-4 loop are involved in bacterial STING oligomerization ${ }^{12}$. Our study here provides a direct structural account for why mutating these predicted residues in SfSTING prevented its oligomerization into long filament ${ }^{12}$. For example, replacement of the 3-4 loop (residues 275-282) with a short linker in SfSTING probably disrupt the extensive hydrophobic interaction in the dimer-dimer interface ${ }^{12}$. Interestingly, the 3-4 loop (residues 181-193) is missing in both monomers of the FsSTING structure, suggesting that the cGAMP-bound FsSTING could not form proper oligomers, or the crystallization condition prevented the protein from oligomerization, and the 3-4 loop was too flexible to be seen in crystal structure ${ }^{12}$. Furthermore, changing A309 (equivalent to F303/F302 in $\alpha 5$ helix of PcSTING/ MySTING) to a positively charged arginine in SfSTING probably caused repulsion or steric clash in the surrounding residues in helix $\alpha 2^{12}$. However, the predicted interface (residues T200-N204 
a

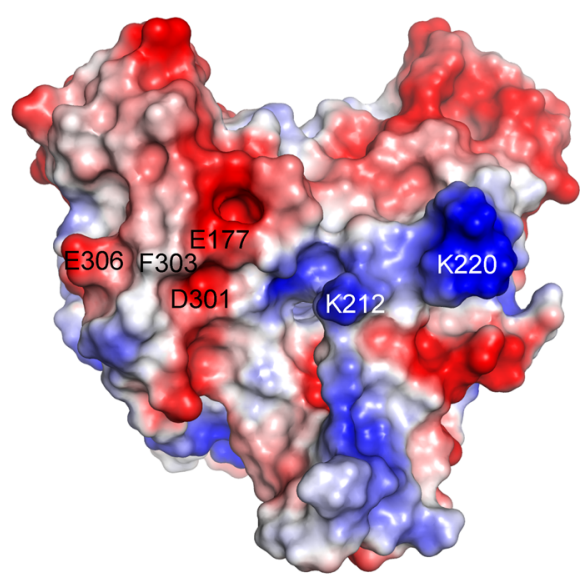

C

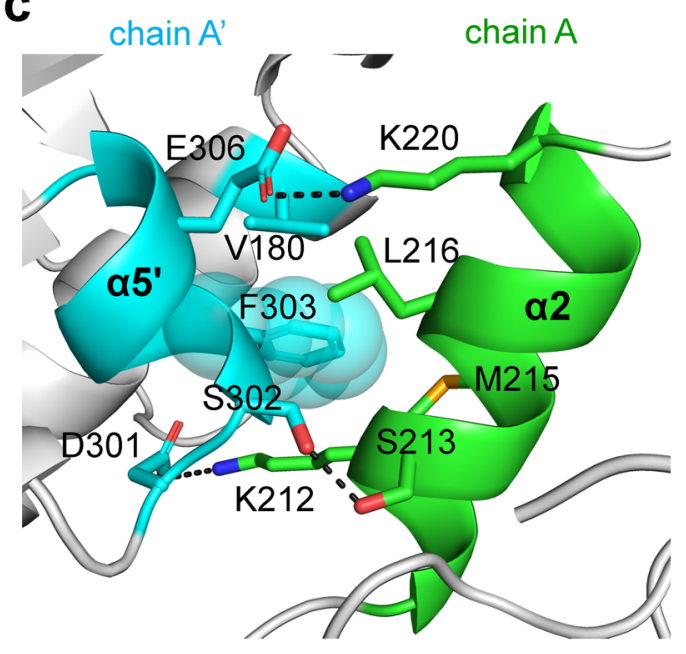

e

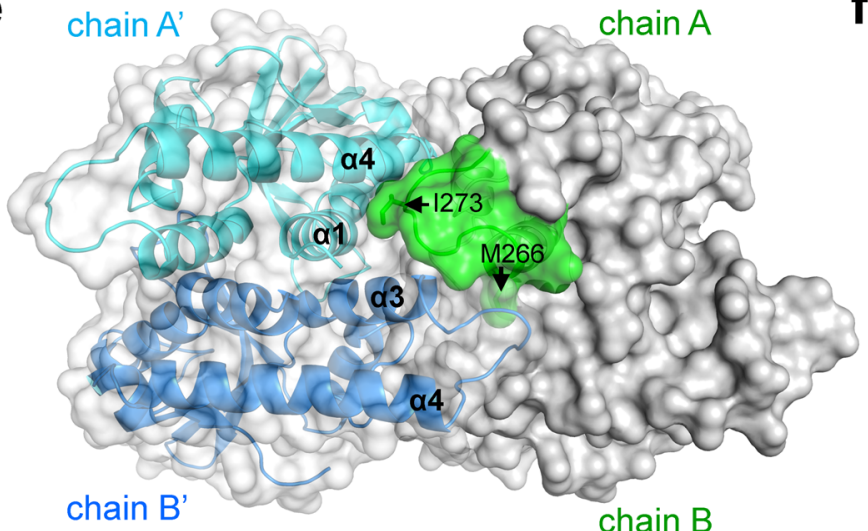

b chain B' chain B

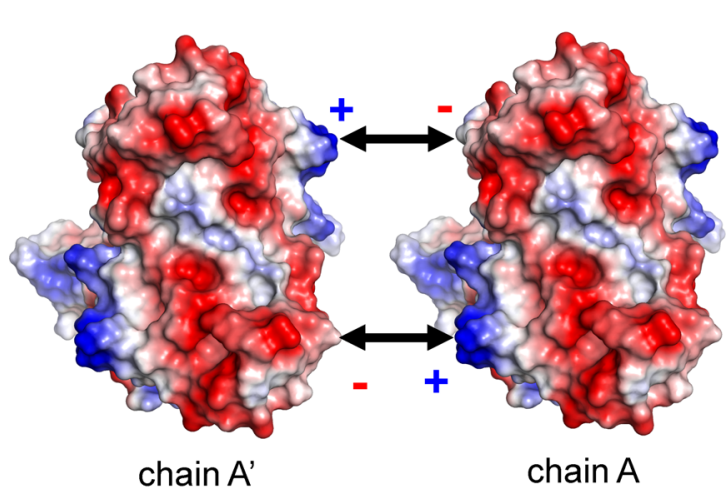

d
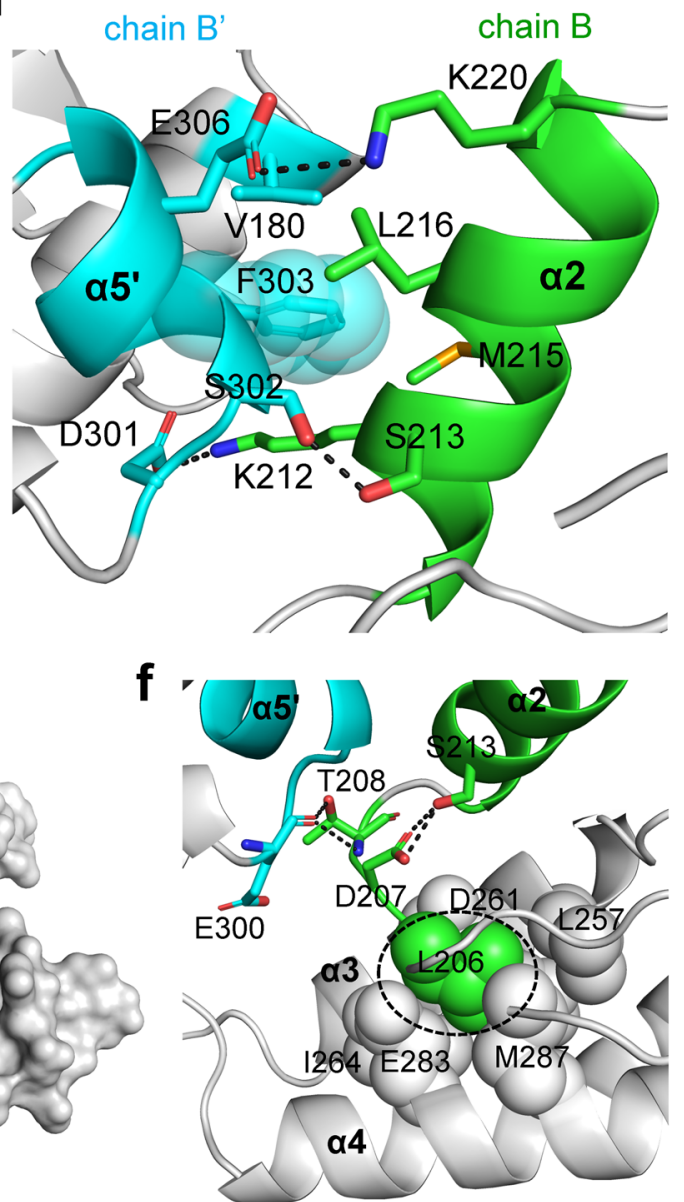

Fig. 6 The oligomerization mechanism of PcSTING revealed by crystal packing. a The electrostatic potential surface of a PCSTING dimer. The surfaces are colored in blue for positive potential $(10 \mathrm{kcal} / \mathrm{mol})$, red for negative $(-10 \mathrm{kcal} / \mathrm{mol})$, and white for neutral. The residues involved in electrostatic interaction and hydrophobic stacking are indicated. $\mathbf{b}$ Binding scheme of dimer-dimer interaction of PCSTING shows that the positively charged patch on one side is complementary to the negatively charged patch on the other side, constituting a basis for filament formation of bacterial STING. c, $\mathbf{d}$ Detailed view of the oligomerization interface between (c) chain $A$ and chain $A^{\prime}$ or (d) chain $B$ and chain $B^{\prime}$ of PCSTING. The chain A/B of the PcSTING dimers in (b). The interacting residues are shown as sticks. The $\mathrm{H}$-bond and ionic bonds are indicated in black dashed lines. To emphasize the stacking interaction, the residue F303 is highlighted by showing its sphere model. e Surface representation of the PCSTING tetramer. The $\alpha 3-\alpha 4$ loop (green, residues 267-276) from chain A of one PCSTING dimer makes extensive hydrophobic interaction with $\alpha 1$ and $\alpha 4$ helix of chain $A^{\prime}$ (cyan) and another $\alpha 3-\alpha 4$ loop of chain $B^{\prime}$ (blue) of the adjacent PCSTING dimer. The side chains of M266 and 1273 that participate in oligomerization are indicated by black arrows. $\mathbf{f}$ The predicted oligomerization interface (residues D119-S123) in FsSTING corresponds to the loop region before $\alpha 2$ helix in PCSTING. Hydrophobic-interacting residues are shown as spheres and hydrophilic interacting residues are shown as sticks. The conserved leucine residue (L206 in PCSTING) that was mutated to arginine and prevented the filament formation in SfSTING is highlighted by a black dashed circle. All the $\alpha$ helixes are labeled in bold fonts. 
in SfSTING) does not directly participate in oligomerization, but instead self-stabilizes the conformation of helix $\alpha 2$ by conserved D207/D204 and helix $\alpha 3$ and $\alpha 4$ by L206/L203 in our PcSTING/ MySTING structures (Figs. 1f and $6 \mathrm{f}$ and Supplementary Fig. 8f). Mutating L201 of SfSTING (equivalent to L206/L203 of PcSTING/MySTING) to arginine may destabilize the tertiary structure, thus hindering its oligomerization. Furthermore, single and double mutants of MyTIR-STING including K209E, F302A, F302R, K209E/F302A, and K209E/F302R were constructed in order to investigate their effects on the dimer-dimer interface. However, the mutations appeared to result in aggregation of improperly formed c-di-GMP bound MyTIR-STING oligomers, which might be treated as a waste by the host cell and degraded. Because none of these mutated proteins could be obtained in an intact form for in vitro study, as an alternative, we tested a comparatively stable mutant K209E/F302R for in vivo activity. This double mutant showed nearly no toxicity to $E$. coli cells, which probably resulted from the instability and degradation of the protein (Supplementary Fig. 9a, b).

\section{Discussion}

In this study, we determined high-resolution structures of PcSTING and MySTING in complex with co-purified endogenous c-di-GMP and clearly demonstrated their strong selectivity for c-di-GMP. The structures consistently elucidate the precise binding mode of c-di-GMP to the bacterial STING, which has been further validated by ITC experiments (Fig. 2a-d and Supplementary Fig. 5a). Changing the guanine base to adenine will substitute the $\mathrm{O}^{6}$ atom (H-bond acceptor) with the $\mathrm{NH}_{2}$ group (H-bond donor), which would result in a significant steric clash with the guanidine group of R233 in PcSTING (R230 in MySTING) and loss of several hydrogen-bonding interactions (Fig. 2a, c). Indeed, the ITC experiments demonstrated that the binding affinity of 3'3'-cGMAP to MySTING is about 127-fold weaker than that of c-di-GMP (Supplementary Fig. 5a, c). Furthermore, changing c-di-GMP to c-di-AMP completely aborted the interaction with MySTING (Supplementary Fig. 5d).

As mentioned earlier, the R153 of FsSTING dimer (equivalent to $\mathrm{Y} 235 / \mathrm{Y} 233$ in PcSTING/MySTING) could either nonspecifically base stack with guanine base or make hydrogen bonds to the Hoogsteen edge of guanine base, providing the structural explanation for the recognition of 3' ''-cGAMP by bacterial STING (Fig. 2e). In fact, the bacterial STING family proteins can be grouped into two classes based on the difference in the identified conserved motifs in the $\beta$-strand lid, which probably dictate their ligand specificity (Fig. 7). Class I bacterial STING proteins have conserved $\mathrm{RX}(\mathrm{Y} / \mathrm{F})$ motif in the $\beta$-strand lid and utilize the four-layer stack interaction to bind specifically to c-di-GMP as revealed by $P c S T I N G$ and $M y S T I N G$ structures in this study. Class II bacterial STING proteins have conserved RXR motif in the $\beta$-strand lid as exemplified by the solved FsSTING structure which preferentially recognized 3',3'-cGAMP. Interestingly, judging by their positions in the phylogenetic tree, the evolution of bacterial STING proteins has branched into two major categories at an early stage, with Class I on one side and Class II on the other. (Supplementary Fig. 10). According to our classification, SfTIR-STING belongs to Class I STING based on its ${ }^{234} \mathrm{RXF}^{236}$ motif and its functional preference for c-di-GMP over 3'3'cGAMP has been validated by a previous study, which further support our findings ${ }^{12}$. In addition, our structural data and bioinformatic analysis (Fig. 2a, c and Supplementary Fig. 4) also provide an explanation for why mutating the base specificitydetermining residue R234 of SfSTING (equivalent to R233/R230 to PcSTING/MySTING) to alanine abolish the stringent specificity for c-di-GMP ${ }^{12}$. Although the functional data interpreting the ligand specificity of Class II bacterial STING is currently absent, the only available crystal structure, FsSTING, revealed a unique asymmetric recognition of 3'3'-cGAMP, which is distinct from the symmetric four-layer stack binding mode of c-di-GMP by Class I STING. Moreover, the functional role of conserved arginine residues in the $\beta$-strand lid of Class II bacterial STING proteins are more closely related to eukaryotic STING, which utilizes two conserved arginine residues (R232 and R238 in hSTING) to recognize the phosphate backbone instead of basespecific interactions (Fig. 7). Therefore, we propose that Class II bacterial STING, which preferentially recognizes 3'3'-cGAMP, is in the evolutionary transition from c-di-GMP-dependent signaling in prokaryote (mediated by Class I STING) to 2'3'-cGAMPdependent signaling in eukaryote (Fig. 7). The functional role of the conserved arginine residues in $\beta$-strand lids changes from base recognition (Class I bacterial STING) to base stacking (Class II bacterial STING) and phosphate backbone recognition (metazoan STING), accounting for the difference in CDN selectivity. This evolution-based model suggests a relationship between STING proteins in different kingdoms of life based on the structural and mechanistic insights provided in this study.

In addition to the differences in the ligand-recognition mode, Class I STING also differ from Class II STING in the tertiary structure and probably the quaternary structure. In comparison with FsSTING (Class II), PcSTING and MySTING (Class I) show an additional small helix $\alpha 5$ and a shorter and differently oriented helix $\alpha 2$ (Fig. 8a). As mentioned above, interactions between helix a5 and $a 2$ mediate the oligomerization of Class I STING. However, helix a5 is absent in Class II STING. Instead, the end of helix $a 4\left({ }^{217} \mathrm{QENNL}{ }^{221}\right)$ may interact with the beginning of helix a2 $\left({ }^{119}\right.$ DELTS $\left.^{123}\right)$ in FsSTING based on the model using chicken STING tetramer as a guide ${ }^{12}$ (Fig. 8a). In addition, helices $\alpha 3$ and a 4 of FsSTING adopt different orientations when compared with PCSTING, and thereby the oligomerization mediated by the 3-4 loop may differ between them (Fig. 8b). Moreover, the conserved residues mediating oligomerization in Class I STINGs are distinct from those in Class II STINGs (Fig. 8c, d). For example, the conserved lysine residue in Class I STINGs (K212 in PcSTING) at the beginning of helix $\alpha 2$ that forms ionic bonds is absent in Class II STINGs (Fig. 8c, d). The distribution of hydrophilic and hydrophobic residues of helix $\alpha 2$ in Class I STINGs is also different from that in Class II STINGs, which probably leads to different oligomerization interfaces among them (Fig. 8c, d). The previous study has demonstrated that cyclic dinucleotide recognition controls bacterial STING oligomerization and the activation of the adjacent effector domain ${ }^{12}$. Therefore, the differences in primary protein sequences and the tertiary structures suggest that the specific ligand-recognition mode may have coevolved with the oligomerization mechanism, resulting in two distinct classes of bacterial STING proteins (Fig. 7). Indeed, the c-di-GMP or 3'3'-cGAMP induced $\beta$-strand lid closure and subsequent conformational changes that oligomerize Class I or Class II STING into active, long filaments are different. C-di-GMP induced the full closure of $\beta$-strand lids that cover the CDN binding pocket and form extensive contact mediated by $\pi-\pi$ stacking interaction between $\mathrm{H} 238 / \mathrm{H} 235$ and $\mathrm{Y} 235 / \mathrm{Y} 232$ in PcSTING/MySTING and additional H-bonding interaction between E225 and N234 in PcSTING (Supplementary Fig. 11a, b). On the contrary, the 3'3'-cGAMP-bound FsSTING showed a loosely packed $\beta$-strand lid mediated by only the base-stacking interaction between F92, R153, and the purines of 3'3'-cGAMP instead of direct interaction between residues on strand $\beta 2$ and $\beta 3$ of FsSTING dimer (Supplementary Fig. 11c) ${ }^{12}$. Superimposition of apo and ligand-bound forms of bacterial STINGs further reveals that structural changes in helices $\alpha 1$ and $a 4$ of PcSTING and FsSTING are accompanied by induced $\beta$-strand lid closure 
a

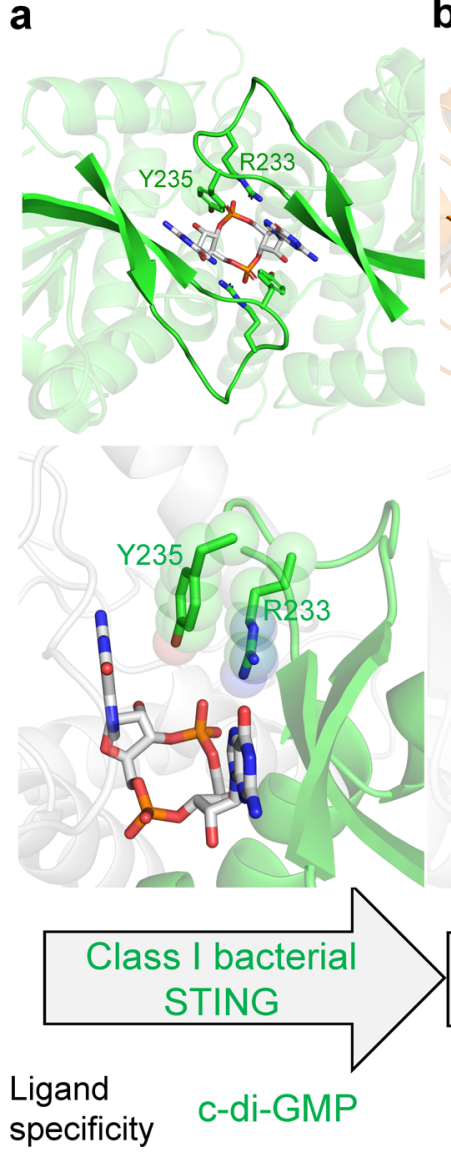

b

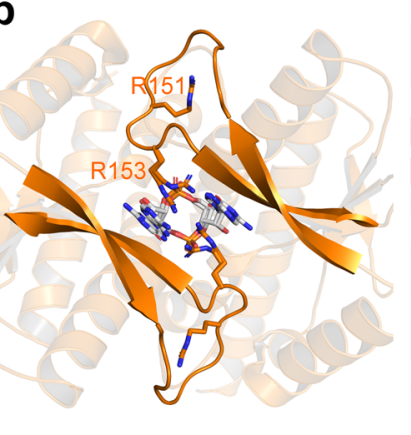

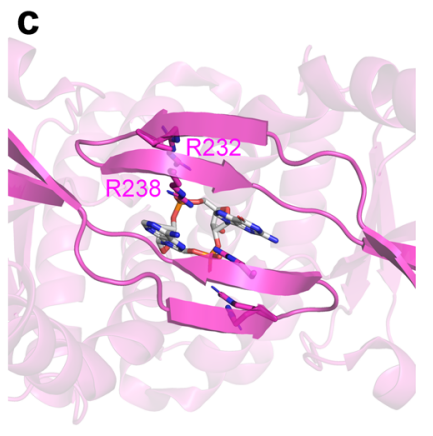
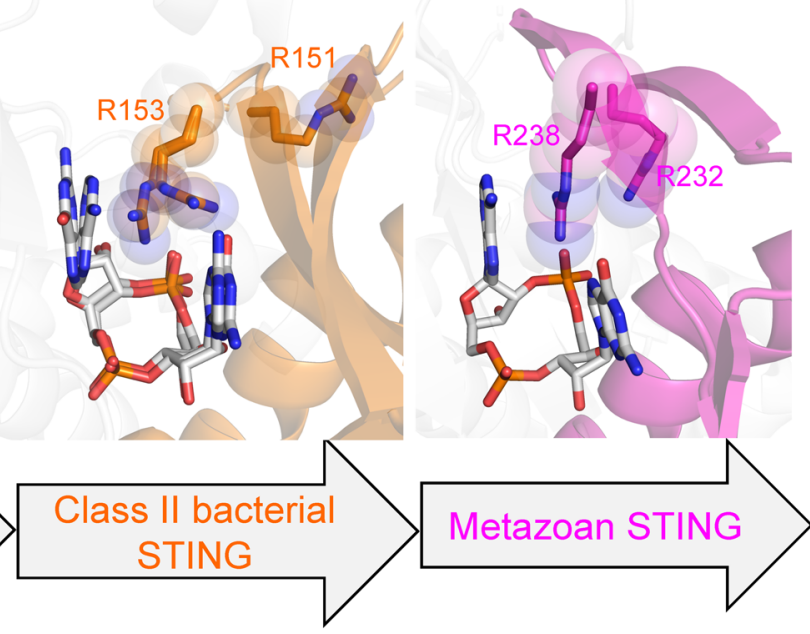

3'3'-cGAMP

2'3'-cGAMP

Fig. 7 Structural comparison of bacterial and metazoan STING suggests a model for the evolution from c-di-GMP based signaling in prokaryotes to the 2'3'-cGAMP-dependent signaling in eukaryotes. The enlarged view of ligand-binding site and $\beta$-strand lid region of (a) PcSTING-c-di-GMP (green), (b) FsSTING-3'3'-cGAMP (orange, PDB: 6WT4), and (c) human STING-2'3'-cGAMP complexes (magenta, PDB: 4KSY). The cyclic dinucleotide ligands are shown as white sticks. The class I bacterial STING has conserved RX(Y/F) motif (R233-X-Y235 in PcSTING) in lid region and specifically recognizes c-diGMP by four-layer stacking interaction. The class II bacterial STING has conserved RXR motif in the $\beta$-strand lid (R151-X-R153 in FsSTING), but recognizes $3^{\prime} 3^{\prime}$-cGAMP asymmetrically. The conserved arginine residues of metazoan STING mainly recognize the $2^{\prime}-5^{\prime} / 3^{\prime}-5^{\prime}$ phosphodiester linkage of $2^{\prime} 3^{\prime}$-cGAMP instead of the nucleobases. The class II bacterial STING is more closely related to the metazoan STING and is proposed to be acquired into the ancestor of early metazoan.

upon c-di-GMP and 3'3'-cGAMP binding, respectively (Supplementary Fig. 12a, b). The conserved phenylalanine residue of $a 5$ helix in Class I STING (F303 in PcSTING) is stabilized by the terminal residues of helix $\alpha 1$ (V180 and T181 in PcSTING) while the corresponding residue L221 in the loop connecting helix a4 and strand $\beta 5$ of FsSTING is stabilized by the residues F94 and I97 in the middle of helix al (Supplementary Fig. 12c, d). Consequently, the differences in cyclic dinucleotide recognition, primary sequence conservation, and the intra- and intermolecular interactions of oligomerization interfaces together result in distinct cyclic dinucleotide-dependent oligomerization between Class I and Class II bacterial STING proteins.

During infection, the innate immunity sensor STING in mammalian cells detect the bacterial CDNs, such as cyclic diGMP and cyclic di-AMP, as pathogen-associated molecular patterns (PAMPs) and activate the downstream immune responses ${ }^{23,24}$. Until now several human STING structures complexed with c-di-GMP have been solved ${ }^{17-20,25}$. All of them recognize c-di-GMP symmetrically with conserved residues Tyr167 forming $\pi-\pi$ stack interaction with guanine nucleobases, which are similar to the base-stacking roles of F92/F171 in FsSTING/PcSTING structures ${ }^{12,17-20,25}$. In particular, one of the c-di-GMP bound hSTING structures (PDB code: 4F5D) revealed a four-layer stack binding mode for c-di-GMP, consisting of
Y167/Gua/R238/Y240 stacking interaction which is similar to the Phe/Gua/Tyr/Arg stacking interaction in PcSTING and MySTING structures identified in this study ${ }^{17,26}$. However, R238 in the four-layer stack of hSTING contacts with the phosphodiester linkage of c-di-GMP instead of the guanine nucleobase as do R233/R230 in PcSTING/MySTING ${ }^{17}$. Adaption of STING proteins for recognizing different cyclic dinucleotides changed the functional role of arginine residues in the $\beta$-strand lid, but the common mode of stacking interaction may be conserved during evolution.

\section{Methods}

Cloning, expression, and purification of bacterial STING proteins. The fulllength gene fragments of TIR-STING proteins from Prevotella corporis (GenBank ID: KXA32418.1) and Myroides sp. ZB35 (IMG Gene ID: 2719779365) were each optimized with E. coli codon usage and synthesized by Genomics BioSci \& Tech company (Supplementary Table 3). The full-length TIR-STING and C-terminal STING domains were subcloned into pSol-MBP or pSol-SUMO vectors to generate target proteins with $\mathrm{N}$-terminal $\mathrm{His}_{6}-\mathrm{MBP}$ or $\mathrm{His}_{6}$-SUMO tag according to the manufacturer's instructions (Expresso ${ }^{\circ}$ Solubility and Expression Screening System, Lucigen). Each of the constructs was transformed into E. coli BL21(DE3) cells and grown in $\mathrm{LB}$ medium at $37^{\circ} \mathrm{C}$ for overnight. For large-scale expression, bacterial cultures were induced with $0.2 \% \mathrm{~L}-$ rhamnose until an $\mathrm{OD}_{600}$ of $0.6-0.8$ was attained and incubated at $16^{\circ} \mathrm{C}$ for $16-20 \mathrm{~h}$. For overexpression of TIR-STING proteins, $30 \mathrm{mM}$ nicotinamide was additionally added to the culture mediums to suppress the cell toxicity caused by $\mathrm{NAD}^{+}$cleavage activity of TIR domain. Cell 
a

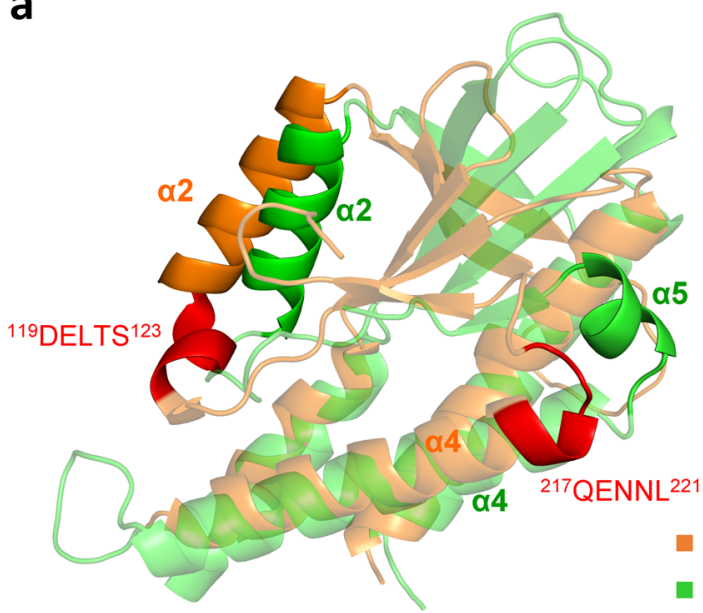

b

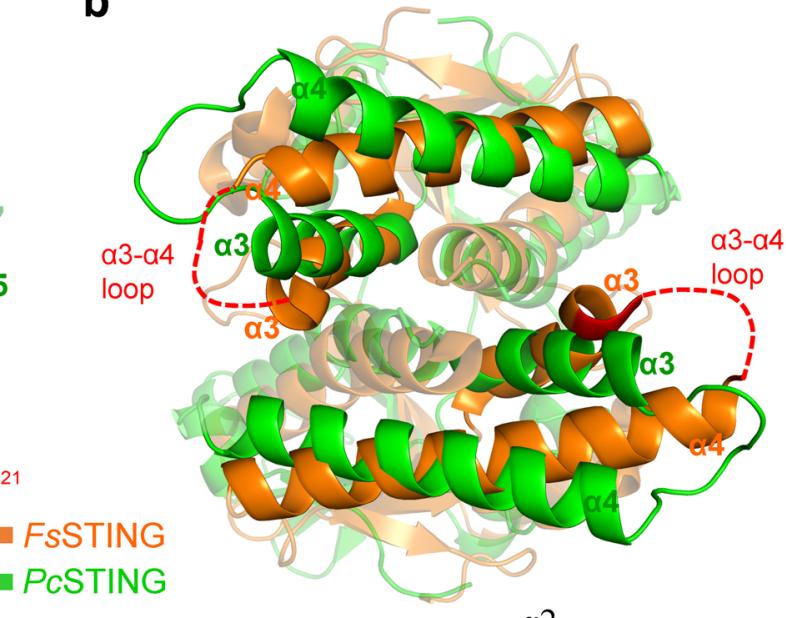

$\alpha 2$
C

PCSTING

BUSTING

MySTING

ChSTING

PCSTING

BuSTING

MySTING

ChSTING

PCSTING

BUSTING

MySTING

ChSTING

d

FsSTING

RaSTING

CgSTING

CSSTING

FsSTING

RaSTING

CgSTING

CSSTING

FsSTING

RaSTING

CgSTING

CSSTING
LPSTVIAISYFEGFVKLAAEWIVTEMPTTEIDGKTYTSGKLYIKMPETLDTDIKKSAMLF LPSTVLAISYFEGFVKLAAEWLTENTPGLEIDGTQYDKGILKIVMPDSLDADIKKCAMLY LPSTVLAIGYFENLVNIICESL-NMLPKLEVSGKEYKKFKFTIVIPKDLDANIKKRAKIY LPSTVLAIGYYENFVSTVCDAL-HSLPTIKLNGIEYKDFVFNIIIPNDLDADIKRRAQIY

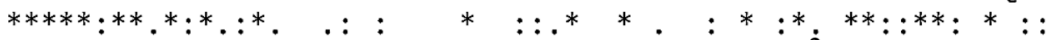
$\frac{\alpha 2}{\text { YKKOGLNETOMSTNHRNYPIHIVSK--EEGDTLEVYDMPTILSGIDKAIDMYFRVGHIGK }}$ YKKLGLKEAKIDTKQRSYPIHFATK--DGEDSLEIYDMPTILTGIDKAIDMYFRVGHIGK FKOKSLIEIEIPTSSRNYPIHIQFDENSTDDILHLYDMPTTIGGIDKAIEMFMRKGHIGK FKKMDIHEVKIDTNGRSFPLYLQIDEENSGDVAVLYDMPTTLGGIDKAIEMYMKKGHIGK

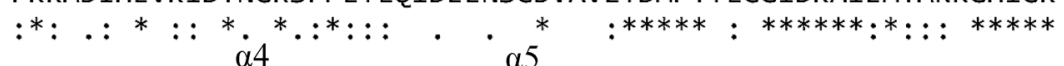
TTEQQLAEDNEMNNFKRVLQLLINEDSFCRECVEIL- - - 154 KIEQELAEENEMNNFRRVLOLLINEDAFCRECVVII--- 154 TDQQKLLEERELRNFKTTLENLIATDAFAKEMVEVIIEE 158 TSQQQLLEERELRNFKTTLINLINNNSFTKTFVKVIEE - 157 . :****:.*:.**: .* ** : :* : * : :
60

60

59

59

118

118

119

119

FIFPAITQIKENGEVNPK - IC - - - - - - IYKPKHFDELTSTNIDMIKAELTNKKYNLSE FVKPTCLHIIQNGGIQDDDGTKYENSTIKIIIPQKLTTDVNSQFQTLKKSFQTK - - - - K FIKPTCSHIINNGGLLDKNGYIYKKCTIKIIIPKKLTSDVNSQFQRIKAKIETK-- - - E FIKPTCTHLVNNDGL-DDEGTKYKECKLKIIIPERLTTDVNLQFQNIKRKFSLK- - - - $\mathrm{K}$

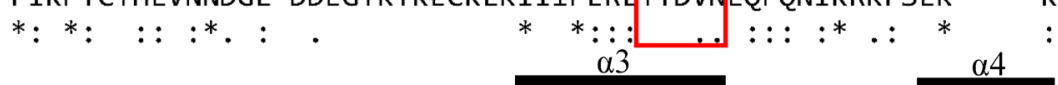
INLSLKGA-RARDILTLNKKSKIHSYFDFPNTLLSLYSYVDFKIASSNNNSSELKKKKFV LTFDYLGRPRNIDVETLIQDG-KLYVIDFPTVLSGI-NYAISNLLPNDFNSM SDDYELIL LSFEYLGRPRNINVEIIAEDG-EVMI IDFPTILSGI-NYAISNLLPQDFNSM SVDYEAIL LSFDYAGRPRNIEVEAIINDN-KVFI IDFPTVLSGI-NYAISNL LPNDFNKM SPDYDSII

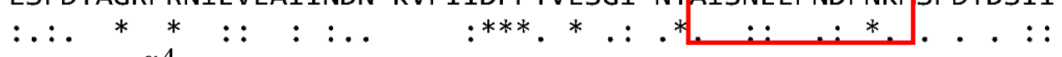

51 55 55 54

Fig. 8 The differences in oligomerization interface between Class I and Class II bacterial STING. a The predicted oligomerization interface at the beginning of $\alpha 2$ helix and the end of $\alpha 4$ helix of FSSTING (orange, PDB: 6WT4) are indicated and colored in red. By contrast, PCSTING (green) protomer uses the $\alpha 5$ helix to interact with the whole $\alpha 2$ helix of the adjacent PCSTING protomer. $\mathbf{b}$ The PCSTING and FsSTING dimers are superimposed. The orientation of $\alpha 3$ and $\alpha 4$ helix and thereby the predicted $\alpha 3-\alpha 4$ loop of FsSTING (red dashed lines) are different from those of PCSTING. c Multiple sequence alignment of four representatives of Class I bacterial STING proteins. $\mathbf{d}$ Multiple sequence alignment of four representatives of Class II bacterial STING proteins. The predicted oligomerization interfaces in FsSTING are highlighted by red frames.

pellets were resuspended in buffer A ( $50 \mathrm{mM}$ Tris- $\mathrm{HCl} \mathrm{pH} 8.0,500 \mathrm{mM} \mathrm{NaCl}, 10 \%$ glycerol, $1 \mathrm{mM}$ TCEP, $1 \mathrm{mM}$ phenylmethanesulfonyl fluoride, $5 \mathrm{mM}$ imidazole), lysed using sonication, and centrifuged at $4^{\circ} \mathrm{C}, 25,000 \times g$ for $30 \mathrm{~min}$. The clarified supernatant was loaded onto a Ni-NTA column and the recombinant protein was eluted with linear imidazole gradient using ÄKTA ${ }^{\mathrm{mt}}$ chromatography system
(Cytiva). The $\mathrm{N}$-terminal $\mathrm{His}_{6}-\mathrm{MBP}$ or $\mathrm{His}_{6}$-SUMO tag was then removed by incubating the eluted recombinant protein with TEV protease at $4{ }^{\circ} \mathrm{C}$ for $24-48 \mathrm{~h}$ during dialysis against buffer $(25 \mathrm{mM}$ Tris $8.0,100 \mathrm{mM} \mathrm{NaCl}, 1 \mathrm{mM}$ DTT, $0.5 \mathrm{mM}$ EDTA, $2 \%$ glycerol). After TEV cleavage, the TIR-STING or STING domain proteins were further purified by size-exclusion chromatography using 16/60 
Superdex 200 increase column equilibrated with buffer containing $20 \mathrm{mM}$ Tris $\mathrm{pH}$ $8.0,200 \mathrm{mM} \mathrm{NaCl}, 5 \%$ glycerol, $1 \mathrm{mM}$ TCEP. Site-directed mutagenesis of PcTIRSTING, MyTIR-STING and MySTING was conducted using PfuUltra HighFidelity DNA Polymerase (Agilent). The selenomethionine (SeMet) labeled PcSTING was purified with the same protocol of the native protein. All the primers for cloning and site-directed mutagenesis used in this study are summarized in Supplementary Table 4.

Crystallization, data collection, and structural determination. The optimal protein concentrations for crystallization of PcSTING and MySTING, determined by Pre-Crystallization Test (Hampton), were $6.5 \mathrm{mg} / \mathrm{ml}$ and $4 \mathrm{mg} / \mathrm{ml}$, respectively. Initial crystallization screening was conducted at $4{ }^{\circ} \mathrm{C}$ using sitting-drop vapordiffusion method. The crystals of native PcSTING and $M y$ STING were grown in $0.1 \mathrm{M}$ HEPES $\mathrm{pH}$ 7.5, 0.2 M calcium chloride dihydrate, 28\% v/v PEG 400 and in $0.1 \mathrm{M}$ sodium cacodylate trihydrate $\mathrm{pH} 6.5,0.2 \mathrm{M}$ sodium acetate trihydrate, $15 \%$ w/v PEG 8000, respectively. The crystals of SeMet-labeled PcSTING were grown in $0.1 \mathrm{M}$ sodium cacodylate trihydrate $\mathrm{pH} 6.5,0.2 \mathrm{M}$ sodium acetate trihydrate, $18 \%$ w/v polyethylene glycol 8000 using the native PcSTING crystals as seeds ${ }^{27}$. All the protein crystals were cryoprotected with reservoir solution plus $25 \%$ glycerol before flash vitrification in liquid nitrogen. The X-ray diffraction data were collected and processed by using HKL2000 software at the National Synchrotron Radiation Research Center (NSRRC) in Taiwan ${ }^{28}$. Experimental phases of SeMet-labeled PcSTING were obtained by multi-wavelength anomalous dispersion (MAD) method using PHENIX software ${ }^{29}$. The three-dimensional model of SeMet-labeled PcSTING was then refined and rebuilt iteratively using PHENIX and $\mathrm{COOT}^{29,30}$. The structure of MySTING was determined by molecular replacement using the SeMet-labeled PcSTING structure as a searching template. All the data collection, phasing, model building, and refinement statistics are summarized in Supplementary Tables 1 and 2 . All the figures that depict three-dimensional structures of protein and ligand were prepared by using $\mathrm{PyMOL}^{31}$.

NAD ${ }^{+}$cleavage activity assay. To test the catalytic activity of full-length TIRSTING protein, we employed a fluorescent NAD derivative, $\varepsilon$-NAD, for enzyme activity assay ${ }^{32}$. The reaction mixture containing $500 \mu \mathrm{M} \varepsilon$-NAD, different cyclic dinucleotides (c-di-AMP, c-di-GMP, 3'3'-cGAMP and c-di-UMP) at the indicated concentration in $20 \mathrm{mM}$ HEPES $\mathrm{pH} 7.5,100 \mathrm{mM} \mathrm{KCl}$ was prepared first. The purified protein at a final concentration of $0.5-1 \mu \mathrm{M}$ was added to initiate the reaction. The fluorescence signals were monitored continuously for $1 \mathrm{~h}$ at the emission wavelength of $410 \mathrm{~nm}$ after excitation at $300 \mathrm{~nm}$. The changes of relative fluorescence units at different time points were recorded. For kinetic analysis of $M y$ TIR-STING protein, the initial velocity of the reaction was determined using the data from the first $300 \mathrm{~s}$.

Protein toxicity analysis in E. coli. The full-length $M y$ TIR-STING, $P c$ TIR-STING and the mutant constructs were cloned into pET21 vector for generating $\mathrm{C}$-terminal $\mathrm{His}_{6}$-tagged proteins or $\mathrm{pSol}$-MBP vector for generating N-terminal MBP-tagged proteins and transformed into E. coli BL21(DE3) or C43(DE3) cells. The cells carrying each expression plasmid were first inoculated at $37^{\circ} \mathrm{C}$ in $\mathrm{LB}$ broth overnight and then diluted by fresh medium to an $\mathrm{OD}_{600}$ of about $0.1-0.2$. Overexpression of the TIR-STING proteins was induced by the addition of $0.5 \mathrm{mM}$ IPTG (for pET21 vector) or $0.2 \%$ L-rhamnose (for pSol-MBP vector) and $30 \mathrm{mM}$ nicotinamide. The values of $\mathrm{OD}_{600}$ were recorded every $15 \mathrm{~min}$ for $2.5 \mathrm{~h}$.

Isothermal titration calorimetry (ITC). In order to analyze the binding affinity of different CDNs to MySTING protein and its variant, nano-ITC (TA Instruments) was used. Purified proteins were first dialyzed overnight against assay buffer containing $20 \mathrm{mM}$ Tris $\mathrm{pH} 8.0,150 \mathrm{mM} \mathrm{NaCl}$. The CDNs were dissolved in the same buffer. Twenty injections of CDNs $(2.5 \mu \mathrm{leach})$ at a concentration of $0.15-0.2 \mathrm{mM}$ were sequentially titrated to 8-50 $\mu \mathrm{M}$ wild-type $M y$ STING or $M y \mathrm{~S}$ TING_Y232R with a stirring speed of $300 \mathrm{rpm}$. The heat of dilution resulting from injecting the $\mathrm{CDN}$ into the assay buffer was subtracted. The final data were then analyzed using NanoAnalyze software (TA Instruments).

Bioinformatic analysis. Structure-based sequence alignment of bacterial STING proteins was generated by $\operatorname{Coot}^{33}$. The phylogenetic tree of bacterial STING proteins was calculated by TTOL $^{34}$.

LC-MS/MS analysis of cyclic di-GMP. An UHPLC system (Ultimate 3000 Dionex, Germany) equipped with a C18 reversed-phase column (Atlantis T3 C18 $3.5 \mu \mathrm{m} 2.1 \times 150 \mathrm{~mm}$; Waters) was coupled with a Q-TRAP $6500^{+}$mass spectrometer (Sciex, USA) with an orthogonal electrospray ionization (ESI) source. For identification of cyclic di-GMP, the targeted mass was set at $\mathrm{m} / \mathrm{z}$ 691.1, and major fragment ions of $\mathrm{m} / \mathrm{z} 152.0, \mathrm{~m} / \mathrm{z} 248.0$ and $\mathrm{m} / \mathrm{z} 540.0$ were observed with collision energy $32-40 \mathrm{eV}$. LC gradient elution was applied from $1 \%$ mobile phase $\mathrm{B}$ (acetonitrile mixed with $0.1 \%$ formic acid) to $40 \% \mathrm{~B}$ in the first $15 \mathrm{~min}$ at $250 \mu \mathrm{l} /$ min flow rate and then increased to $99 \%$ B within 3 min and held at $99 \%$ B for another $3 \mathrm{~min}$. Finally, 1\% B was used for $4 \mathrm{~min}$ to re-equilibrate the column prior to the next injection. The total run time was $25 \mathrm{~min}$ and the mobile phase $\mathrm{A}$ consisted of $0.1 \%$ formic acid. The ion spray voltage was set at $5.5 \mathrm{kV}$, and the curtain gas was 25 psi. The ion source gas 1 and 2 were 50 and $60 \mathrm{psi}$, respectively. The source temperature was set at $550^{\circ} \mathrm{C}$.

Statistical analysis. The data from $\mathrm{NAD}^{+}$cleavage activity assay shown in Fig. $4 \mathrm{~b}$, $\mathrm{c}$ and Supplementary $7 \mathrm{a}, \mathrm{b}$ represent mean \pm standard deviation from three independent replicates.

Reporting summary. Further information on research design is available in the Nature Research Reporting Summary linked to this article.

\section{Data availability}

Data are available within the article and supplementary information. Integrated Microbial Genomes (IMG) database accession and GenBank accession number are listed in the "Methods". The coordinates and structure factors of PcSTING/c-di-GMP and MySTING/c-di-GMP complexes have been deposited in the Protein Data Bank with accession codes 7EBD and 7EBL. Source data are provided with this paper.

Received: 21 April 2021; Accepted: 20 September 2021; Published online: 10 January 2022

\section{References}

1. Safari, F. et al. The interaction of phages and bacteria: the co-evolutionary arms race. Crit. Rev. Biotechnol. 40, 119-137 (2020).

2. Burroughs, A. M., Zhang, D., Schäffer, D. E., Iyer, L. M. \& Aravind, L. Comparative genomic analyses reveal a vast, novel network of nucleotidecentric systems in biological conflicts, immunity and signaling. Nucleic Acids Res. 43, 10633-10654 (2015).

3. Hampton, H. G., Watson, B. N. J. \& Fineran, P. C. The arms race between bacteria and their phage foes. Nature 577, 327-336 (2020).

4. Rostøl, J. T. \& Marraffini, L. (Ph)ighting phages: how bacteria resist their parasites. Cell Host Microbe 25, 184-194 (2019).

5. Maxwell, K. L. Phages fight back: inactivation of the CRISPR-Cas bacterial immune system by anti-CRISPR proteins. PLoS Pathog. 12, el005282 (2016).

6. Pawluk, A., Davidson, A. R. \& Maxwell, K. L. Anti-CRISPR: discovery, mechanism and function. Nat. Rev. Microbiol. 16, 12-17 (2018).

7. Cohen, D. et al. Cyclic GMP-AMP signalling protects bacteria against viral infection. Nature 574, 691-695 (2019).

8. Millman, A., Melamed, S., Amitai, G. \& Sorek, R. Diversity and classification of cyclic-oligonucleotide-based anti-phage signalling systems. Nat. Microbiol. 5, 1608-1615 (2020).

9. Bernheim, A. \& Sorek, R. The pan-immune system of bacteria: antiviral defence as a community resource. Nat. Rev. Microbiol. 18, 113-119 (2020).

10. Cheng, Z. et al. The interactions between cGAS-STING pathway and pathogens. Signal Transduct. Target. Ther. 5, 91 (2020).

11. Gui, X. et al. Autophagy induction via STING trafficking is a primordial function of the cGAS pathway. Nature 567, 262-266 (2019).

12. Morehouse, B. R. et al. STING cyclic dinucleotide sensing originated in bacteria. Nature 586, 429-433 (2020).

13. Essuman, K. et al. TIR domain proteins are an ancient family of NAD(+)consuming enzymes. Curr. Biol.: CB 28, 421-430 (2018). e424.

14. Horsefield, $\mathrm{S}$. et al. $\mathrm{NAD}(+)$ cleavage activity by animal and plant TIR domains in cell death pathways. Science 365, 793-799 (2019).

15. Margolis, S. R., Wilson, S. C. \& Vance, R. E. Evolutionary origins of cGASSTING signaling. Trends Immunol. 38, 733-743 (2017).

16. Mahadevi, A. S. \& Sastry, G. N. Cation- $\pi$ interaction: its role and relevance in chemistry, biology, and material science. Chem. Rev. 113, 2100-2138 (2013).

17. Huang, Y. H., Liu, X. Y., Du, X. X., Jiang, Z. F. \& Su, X. D. The structural basis for the sensing and binding of cyclic di-GMP by STING. Nat. Struct. Mol. Biol. 19, 728-730 (2012).

18. Ouyang, S. et al. Structural analysis of the STING adaptor protein reveals a hydrophobic dimer interface and mode of cyclic di-GMP binding. Immunity 36, 1073-1086 (2012)

19. Shang, G. et al. Crystal structures of STING protein reveal basis for recognition of cyclic di-GMP. Nat. Struct. Mol. Biol. 19, 725-727 (2012).

20. Yin, Q. et al. Cyclic di-GMP sensing via the innate immune signaling protein STING. Mol. cell 46, 735-745 (2012).

21. Cong, X. et al. Crystal structures of porcine STING(CBD)-CDN complexes reveal the mechanism of ligand recognition and discrimination of STING proteins. J. Biol. Chem. 294, 11420-11432 (2019).

22. Zhang, X. et al. Cyclic GMP-AMP containing mixed phosphodiester linkages is an endogenous high-affinity ligand for STING. Mol. Cell 51, 226-235 (2013). 
23. Schaap, P. Cyclic di-nucleotide signaling enters the eukaryote domain. IUBMB life 65, 897-903 (2013).

24. Shaw, N., Ouyang, S. \& Liu, Z. J. Binding of bacterial secondary messenger molecule c di-GMP is a STING operation. Protein Cell 4, 117-129 (2013).

25. Shu, C., Yi, G., Watts, T., Kao, C. C. \& Li, P. Structure of STING bound to cyclic di-GMP reveals the mechanism of cyclic dinucleotide recognition by the immune system. Nat. Struct. Mol. Biol. 19, 722-724 (2012).

26. Chou, S. H. \& Galperin, M. Y. Diversity of cyclic di-GMP-binding proteins and mechanisms. J. Bacteriol. 198, 32-46 (2016).

27. D'Arcy, A., Villard, F. \& Marsh, M. An automated microseed matrixscreening method for protein crystallization. Acta Crystallogr. Sect. D., Biol. Crystallogr. 63, 550-554 (2007).

28. Otwinowski, Z. \& Minor, W. Processing of X-ray diffraction data collected in oscillation mode. Methods Enzymol. 276, 307-326 (1997)

29. Liebschner, D. et al. Macromolecular structure determination using X-rays, neutrons and electrons: recent developments in Phenix. Acta Crystallogr. Sect. D., Struct. Biol. 75, 861-877 (2019).

30. Emsley, P., Lohkamp, B., Scott, W. G. \& Cowtan, K. Features and development of Coot. Acta Crystallogr. Sect. D., Biol. Crystallogr. 66, 486-501 (2010).

31. Schrodinger, L. L. C. The PyMOL Molecular Graphics System, Version 2.3.3 (2019).

32. Pergolizzi, G., Butt, J. N., Bowater, R. P. \& Wagner, G. K. A novel fluorescent probe for NAD-consuming enzymes. Chem. Commun. 47, 12655-12657 (2011).

33. Krissinel, E. \& Henrick, K. Secondary-structure matching (SSM), a new tool for fast protein structure alignment in three dimensions. Acta Crystallogr. Sect. D., Biol. Crystallogr. 60, 2256-2268 (2004).

34. Letunic, I. \& Bork, P. Interactive Tree Of Life (iTOL) v4: recent updates and new developments. Nucleic Acids Res. 47, W256-w259 (2019).

\section{Acknowledgements}

The authors are grateful to NSRRC for beam time allocations and assistance with data collection, and to the Academia Sinica Protein Clinic, funded by Academia Sinica Core Facility and Innovative Instrument Project [AS-CFII-109-107] for the X-ray crystallographic service. This work was supported by grants from the Ministry of Science and Technology in Taiwan (108-2311-B241-001 and 109-2311-B241-001) and China Medical University in Taiwan (CMU109-MF-18 and CMU109-S-07).

\section{Author contributions}

T.P.K., Y.C.W., C.S.Y., and M.H.H. designed and carried out the experiments and acquired and analyzed the data. C.J.C. and Y.F.C. carried out the experiments. Y.C. interpreted the data, wrote the manuscript, and supervised the entire project.

\section{Competing interests}

The authors declare no competing interests.

\section{Additional information}

Supplementary information The online version contains supplementary material available at https://doi.org/10.1038/s41467-021-26583-3.

Correspondence and requests for materials should be addressed to Yeh Chen.

Peer review information Nature Communications thanks Lichuan Gu and the other, anonymous, reviewer(s) for their contribution to the peer review of this work.

Reprints and permission information is available at http://www.nature.com/reprints

Publisher's note Springer Nature remains neutral with regard to jurisdictional claims in published maps and institutional affiliations.

(c) Open Access This article is licensed under a Creative Commons Attribution 4.0 International License, which permits use, sharing, adaptation, distribution and reproduction in any medium or format, as long as you give appropriate credit to the original author(s) and the source, provide a link to the Creative Commons license, and indicate if changes were made. The images or other third party material in this article are included in the article's Creative Commons license, unless indicated otherwise in a credit line to the material. If material is not included in the article's Creative Commons license and your intended use is not permitted by statutory regulation or exceeds the permitted use, you will need to obtain permission directly from the copyright holder. To view a copy of this license, visit http://creativecommons.org/ licenses/by/4.0/.

(C) The Author(s) 2022 